\title{
Melatonin and sleep disorders in patients with severe atopic dermatitis
}

\author{
Andrzej K. Jaworek ${ }^{1}$, Magdalena Jaworek ${ }^{2}$, Krystyna Szafraniec ${ }^{3}$, Anna Wojas-Pelc ${ }^{1}$, Jacek C. Szepietowski ${ }^{4}$ \\ ${ }^{1}$ Department of Dermatology, Jagiellonian University Medical College, Krakow, Poland \\ ${ }^{2}$ Department of Physiotherapy, Faculty of Health Sciences, Jagiellonian University Medical College, Krakow, Poland \\ ${ }^{3}$ Department of Epidemiology and Population Studies, Institute of Public Health, Faculty of Health Sciences, Jagiellonian University \\ Medical College, Krakow, Poland \\ ${ }^{3}$ Department of Dermatology, Venereology and Allergology, Wroclaw Medical University, Wroclaw, Poland
}

Adv Dermatol Allergol 2021; XXXVIII (5): 746-751

DOI: https://doi.org/10.5114/ada.2020.95028

\begin{abstract}
Introduction: Many atopic dermatitis (AD) patients suffer from insomnia. Out of numerous factors associated with sleep disorders, melatonin seems to play a significant role.

Aim: To assess the relation between melatonin concentration and sleep disorders in adult patients with severe and very severe AD.

Material and methods: The study included 36 adult patients with severe and very severe AD and 20 healthy Caucasian volunteers. The severity of skin lesions was assessed by the EASI scale. Skin itch was evaluated by a visual-analogue scale (VAS), and sleep disorders were assessed by the Polish version of the Athens Insomnia Scale (AIS). Serum melatonin concentration of patients and controls was determined by ELISA.

Results: Melatonin concentration in patients with very severe AD was significantly $(p<0.001)$ lower than in patients with severe AD, however, melatonin concentration in the group of AD patients did not differ significantly $(p=0.33)$ from that observed in the control group. There was a significant negative correlation between the concentration of melatonin in the study group and the severity of itching $(R=-0.54, p<0.001)$. The intensity of sleep disorders was significantly higher $(p<0.001)$ in patients with a very severe form of AD compared to patients with severe AD. Moreover, there was a significant negative correlation between melatonin concentration and sleep disorders $(R=-0.67, p<0.001)$.

Conclusions: Our results clearly showed that sleep disturbances are more expressed in very severe AD patients compared to subjects suffering from severe disease. We also suggest that melatonin serum concentration could play a role in the pathogenesis of sleep disturbances in AD patients.
\end{abstract}

Key words: atopic dermatitis, sleep disorders, melatonin, itch.

\section{Introduction}

Atopic dermatitis (AD; atopic eczema - AE) is a chronic, recurrent, common inflammatory skin disease, characterized by periods of exacerbation and remission. The disease manifests itself as an intensive itching accompanied by skin lesions of an eczematous morphology (acute, subacute or chronic), whose location depends on the patient's age $[1,2]$.

It has been noted in recent years that in addition to comorbid atopic diseases, AD is often accompanied by a number of other non-atopic diseases, such as skin infections, cardiovascular diseases, cancer, and particularly often by mental and neurological disorders [3, 4]. A substantial number of AD patients (33-87.1\% of adults and $47-80 \%$ of children) experience sleep difficulties, which exacerbate the adverse effects of the disease on their mental and socio-economic functioning [5]. The most common sleep disorders in AD patients include: difficulties with falling asleep, frequent night awakenings, problems with getting up in the morning, sleepiness during the day, sleep apnoea and even a restless legs syndrome [5-7]. Among various factors associated with sleep disorders the one which seems to be of particular importance is melatonin - a neurohormone which, among other

Address for correspondence: Andrzej K. Jaworek, Department of Dermatology, Jagiellonian University Medical College, 50 Kopernika St, 31-501 Krakow, Poland, phone: + 48694486 112, e-mail: andrzej.jaworek@uj.edu.pl Received: 2.04.2020, accepted: 10.04.2020. 
functions, regulates the circadian rhythm of the sleepwake cycle [8].

\section{Aim}

The aim of the study was to investigate the relationship between melatonin concentration and sleep disturbances in adult patients with severe and very severe AD.

\section{Material and methods}

The study was approved by the Bioethics Committee of the Jagiellonian University and all participants signed informed, voluntary consent to participate in the study. A group of 36 adult patients being in the exacerbation period with childhood-onset $A D$ and 20 healthy Caucasian volunteers were qualified to the study. The characteristics of the study group are presented in Table 1. The diagnosis of the disease was established based on the Hanifin and Rajka criteria [9]; the severity of lesions was based on the Eczema Area and Severity Index (EASI, where mild AD: 1.1-7.0 points, moderate AD: 7.1-21.0 points, severe AD: 21.1-50.0 points, very severe AD: 50.1-72.0) [10]. Furthermore, each participant performed a self-assessment of the severity of the most intensive pruritus during the preceding $24 \mathrm{~h}$ using a Visual-Analogue Scale (VAS, where 0 points: no pruritus, 1-3: mild pruritus, 4-6: moderate pruritus, 7-8: severe pruritus, 9-10: very severe pruritus) [11]. All study participants were asked to complete the validated Polish version of the Athens Insomnia Scale (AIS) consisting of eight questions (each with three answers), which defined the following parameters of sleep during the preceding month: falling asleep, night-time awakenings, early morning awakenings, total sleep duration, quality of sleep, sense of well-being during the day, mental and physical functioning, and sleepiness during the day. The result (0-24 points) $\geq 6$ points indicated sleep disorders [12].

The exclusion criteria were lack of consent, age below 18 years, presence of other inflammatory, pruritic systemic diseases, taking anti-pruritic and immunosuppressive medications (up to 3 months prior to the study) and undergoing phototherapy up to 6 months prior to the study. Other causes of insomnia (psychological and internal) were excluded in all the patients. No participant had taken sleeping pills for up to 3 months prior to the study.

Three millilitres of blood for melatonin testing were taken from AD patients and from the control group. Blood was taken from the ulnar vein at 8.00 am sharp, by the same person under the same circumstances. Serum concentrations of melatonin in the blood of patients and controls were determined by ELISA, using a commercial kit from R\&D System (Minneapolis, USA). Blood samples were left for $2 \mathrm{~h}$ at room temperature to clot, then centrifuged (3500 rpm for $10 \mathrm{~min}$ ) and subsequently the serum samples were frozen and stored at $-80^{\circ} \mathrm{C}$ until assayed.

\section{Statistical analysis}

Nonparametric methods were used in statistical analysis of the results: 1 ) Mann-Whitney $U$ test to compare the distribution of melatonin and the severity of sleep disorders (AIS) in groups with various severity of skin lesions (EASI), 2) Spearman's rank correlation to determine the strength and direction of the relationship between the severity of pruritus and melatonin levels. The level of statistical significance was considered to be $\alpha=0.05$. Calculations were performed by means of Dell Statistica software, version 13 .

\section{Results}

Average serum melatonin concentrations in the group of patients (median: $228.6 \mathrm{pg} / \mathrm{ml}$; min.-max.: 46.9-534.9 $\mathrm{pg} / \mathrm{ml}$ ) and in the control group (median: $230.2 \mathrm{pg} / \mathrm{ml}$; min.-max.: 159.0-538.2 pg/ml) were not statistically significantly different $(p=0.33)$. There were no statistical differences in serum melatonin concentrations between younger and older patients in our study. However, there was a statistically significant difference $(p<0.001)$ in melatonin concentration in patients with $A D$ of different clinical severity (Figure 1). Namely, melatonin concentration in patients with very severe AD (median: $68.9 \mathrm{pg} / \mathrm{ml}$;

Table 1. Characteristics of the study group

\begin{tabular}{lcc}
\hline Parameter & Patients $(n=36)$ & Controls $(n=20)$ \\
\hline $\begin{array}{l}\text { Gender: } \\
\text { Female }\end{array}$ & $21(58 \%)$ & $11(55 \%)$ \\
\hline Male & $15(42 \%)$ & $9(45 \%)$ \\
\hline Age, median (min.-max.) & $40(20-75)$ & $28(23-67)$ \\
\hline EASI (pts), median (min.-max.) & $39.1(25.0-55.4)$ & \\
\hline Severe (21.1-50 pts) & $26(72.2 \%)$ & \\
\hline Very severe (> 50 pts) & $10(27.8 \%)$ & \\
\hline
\end{tabular}




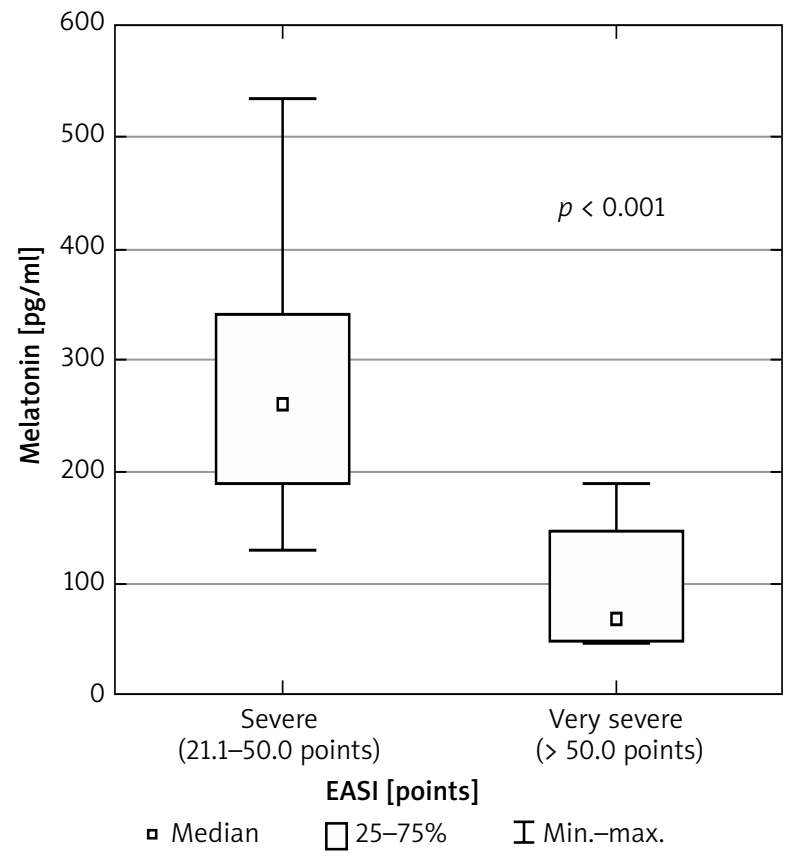

Figure 1. Comparison of melatonin concentration in groups of patients with severe and very severe AD $(p<0.001)$

min.-max.: 46.9-190.5 pg/ml) was significantly lower than in patients with severe AD (median: $260.7 \mathrm{pg} / \mathrm{ml}$; min.-max.: 129.6-534.9 pg/ml).

Moreover, there were significant negative correlations between the serum melatonin level and AD severity and intensity of itch assessed by VAS $(R=-0.54, p<0.001$; $R=-0.64, p<0.001$, respectively) (Figures 2, 3).

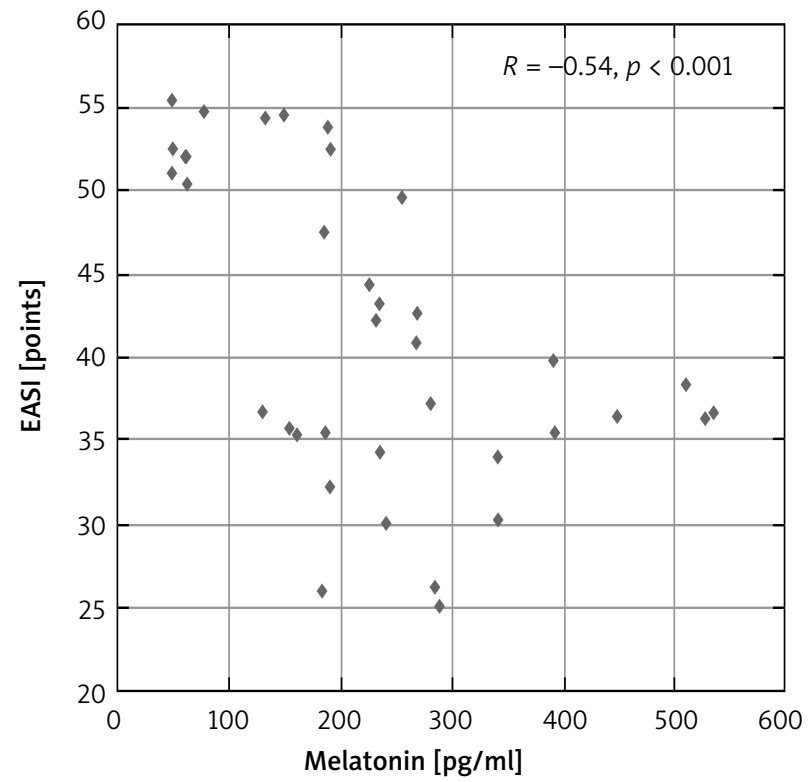

Figure 2. Correlation between melatonin concentration and intensity of $A D$ (EASI points) in the study group of patients
All examined patients (100\%) had sleep disorders according to the AIS (> 6 pts), whereas in the control group not a single individual met the sleep disorders criteria. The median intensity of sleep disturbances in AD patients, assessed by the AIS, was 14 points (min.-max.: 8-17 pts). Sleep disturbances were statistically significantly higher $(p<0.001)$ in patients with very severe AD (median: 16 points; min.-max.: 14-17 pts) compared to patients with severe AD (median: 12 pts; min.-max.: 816 pts) (Figure 4).

Moreover, a statistically significant negative correlation between melatonin concentration and sleep disturbances (according to AIS) was observed in patients with $\mathrm{AD}(R=-0.67, p<0.001)$ (Figure 5$)$.

\section{Discussion}

Even though $A D$ is commonly associated with the child population, the results of an extensive study by Barbarot et al. [13] including the population of people from the USA, Japan, Canada and Europe indicate a significant underestimation of the disease prevalence in the adult population (AD symptoms were observed in as much as $8.2 \%$ of adults). To meet the needs of practising physicians, the Australian Dermatological Society decided in 2019 to develop guidelines for AD diagnosis and therapy in adults [14]. The analysis carried out by Raciborski et al. [15] demonstrated that in Poland AD was the reason for almost 260,000 specialist consultations and 8,000 hospitalisations, which translated to PLN 26.5 million spent by the National Health Fund on AD patient care. The pathogenesis of $A D$ is associated with the interaction of environmental and immunological factors with the over-

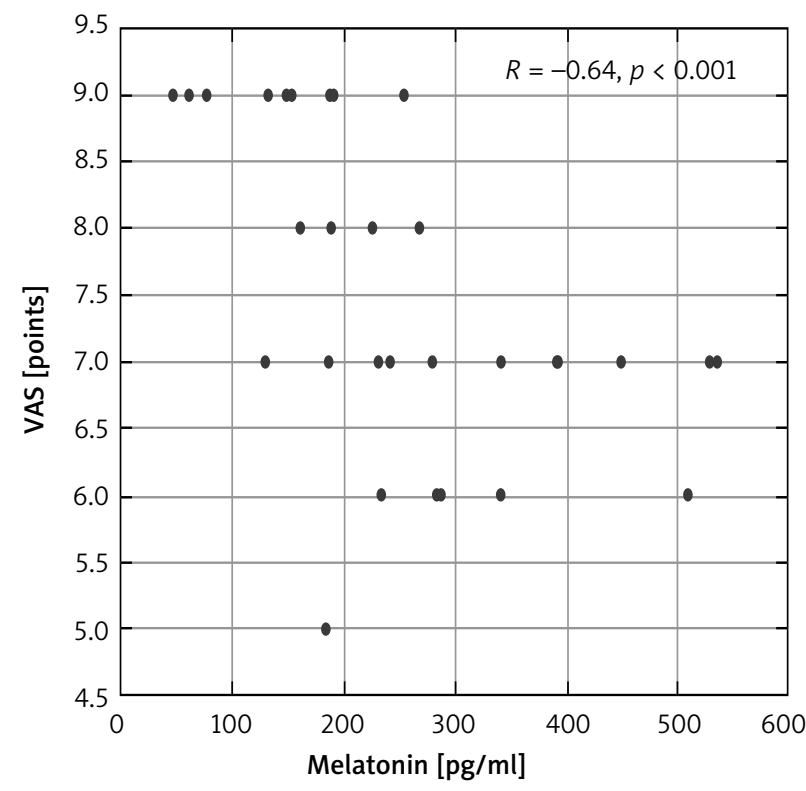

Figure 3. Correlation between melatonin concentration and pruritus (VAS points) in the study group of patients 


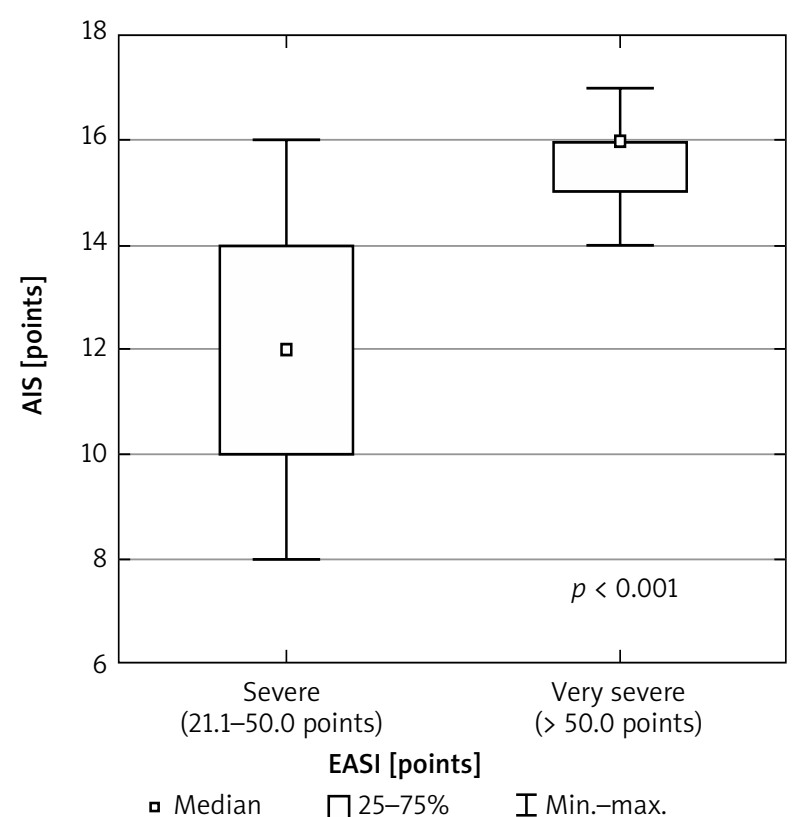

Figure 4. Sleep disorders (according to AIS) in patients with severe and very severe $A D$

lapping skin barrier deficiency; however, mechanisms related to the development of this disease are still the subject of scientific investigations [16].

Sleep is the essential functional state of the central nervous system and its disorders result in unfavourable psychosocial and economic consequences in AD patients (e.g. lower material status, concentration disorders, increased number of visits to the doctor) $[17,18]$.

Our observations indicate a positive correlation between the severity of skin lesions and the intensity of sleep disturbances in AD patients, which corroborates the findings of other authors, albeit obtained in research involving child patients $[19,20]$.

AD disturbs the physiological course of sleep in both rapid eye movement (REM) and non-rapid eye movement (NREM) phases, but the mechanisms underlying sleep disturbances in AD patients are still unexplained. The most frequently mentioned factors contributing to sleep disorders in AD patients include: itch followed by scratching, flares of comorbid atopic diseases, the so-called learned insomnia (also present in the phase of disease remission), elevated concentrations of acetylcholine, norepinephrine and histamine, which disturb the sleep-wake cycle, disorders in the population of inflammatory cells and the factors they secrete in patients' skin, increased dysfunction of the skin barrier at night, and decreased production of melatonin $[5,8]$.

Melatonin (N-acetyl-5-methoxytryptamine), an L-tryptophan metabolite, was first isolated in the 1950s by dermatologist Aaron Lerner of Yale University. Although it is mostly known to be produced by a pineal gland, it is also synthesized in skin and gastrointestinal cells, lymphocytes and mast cells, among others. Melatonin is an

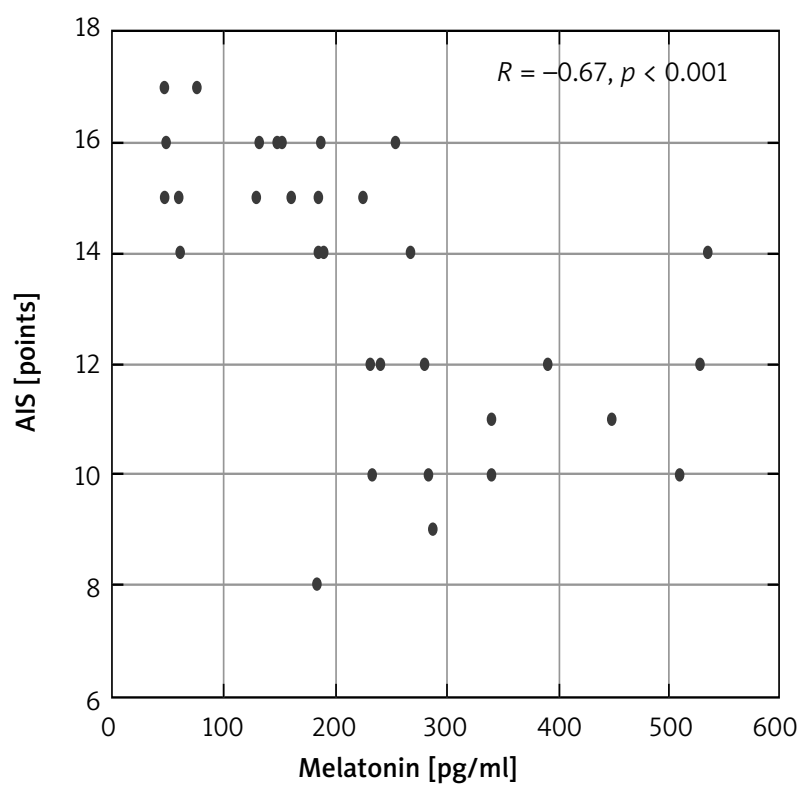

Figure 5. Correlation between melatonin concentration and sleep disturbances (AIS points) in the study group of patients

indoleamine which, due to its amphipathic nature, easily passes through the blood-brain barrier and cell membranes, reaches the peripheral tissues (including skin) and affects the intracellular compartments [21]. It is worth noting that melatonin is produced by keratinocytes and, as an antioxidant, protects the skin against UV damage [5]. The widely recognised antioxidant properties of melatonin are associated with its anti-inflammatory effects (e.g. inhibition of induced NO synthase and cyclooxygenase-2, reduction of NF- $\mathrm{KB}$ and modulation of pro- and anti-inflammatory cytokines) [22]. Since melatonin is released by the pineal gland regularly in the course of the day-night cycle, it is considered to be the regulator of circadian rhythms, with its most important role being the regulation of the sleep-wake rhythm [5, 21-23].

A study by Schwarz et al. [24] from 1988 showed that in a group of 18 children with $A D$, the concentration of melatonin and its daily secretion rhythm were comparable to the control group (40 individuals) only in 4 children. Research of Munoz-Hoyos et al. [25] including the group of 40 children with various stages of AD indicated statistically significant differences in melatonin concentration not only between the group of patients during a flare-up and the control group (the highest melatonin concentration), but also between patients with varying severity of skin lesions. This observation is consistent with the results of our study: in the group of patients with a very severe disease, blood melatonin concentration was significantly lower than in the group of patients with severe AD.

In 2014, Chang et al. [19] having examined 72 AD patients up to the age of 18 , found (based on polysomnographic and actigraphic recordings) that the severity 
of the disease predisposes to sleep disorders (especially in patients with $S C O R A D \geq 48.7$ points). A similar correlation was observed in our patients, however it should be noted that the EASI seems to be more useful for objective assessment of the severity of skin lesions when investigating sleep disorders in AD as it does not contain elements of subjective self-assessment of symptoms by the patient. Moreover, a decreased night blood melatonin concentration in patients described by Chang et al. [19] was statistically significantly associated with higher SCORAD, whereas the concentration of melatonin metabolite (6-sulfatoxymmethylatonin) in urine correlated negatively with the severity of the disease. Other factors that contributed to sleep disturbances in patients included: elevated total IgE concentration, itch and associated scratching as well as allergy to house dust mites and staphylococcal enterotoxins. Another report by the same researchers concluded that melatonin supplementation at the dose of $3 \mathrm{mg} /$ day for 1 month (versus placebo) in a small group of children and adolescents proved to be effective in reducing symptoms (SCORAD; statistical significance) and time needed to fall asleep [26]. Similar conclusions were reached by Iranian authors whose research study included a group of 70 children. The researchers administered melatonin (to 35 children vs. placebo to 35 children) at a dose of $6 \mathrm{mg} /$ day for 6 weeks, which resulted in a statistically significant reduction in the severity of lesions (SCORAD, objective SCORAD) and improvement in sleep parameters (Children's Sleep Habits questionnaire) as well as in total IgE concentration. Interestingly, the treatment did not affect the severity of itching or the inflammation parameters (CRP) [27]. Results of recent studies indicate that melatonin, also due to its multidirectional anti-inflammatory and anti-apoptotic effects, may prove to be a promising therapeutic option in patients with $\operatorname{AD}[28,29]$. Our study revealed a negative correlation between the concentration of melatonin and the severity of sleep disorders and pruritus in adult patients (the lower the melatonin level, the more severe sleep disorders and pruritus). It seems worthwhile to conduct studies on the effect of melatonin supplementation on the course of the disease in adults with AD.

Considering the role of melatonin in the pathomechanism of $A D$, the results of experimental studies should also be mentioned, which showed that administering melatonin to mice with AD significantly reduced neuronal damage and expression of neurotoxic cytokines in the nervous system, which resulted in decreased skin itch and scratching-related damage. After melatonin administration, the IgE and $\mathrm{CRH}$ levels in these animals were also reduced [30].

A 2019 report published by Wroclaw-based authors investigated the impact of itching (according to VAS) on the quality of sleep (according to AIS and Pittsburgh Sleep Quality Index) in a group of 100 adult AD patients (SCORAD: $33.6 \pm 10.7$ points; the control group consisted of 50 people). The results of that study corroborated our findings. There was a statistically significant correlation between the severity of skin lesions and the results of both insomnia scales analysis. The severity of itching was statistically significantly higher in the patient group compared to the control group. The authors suggested using other scales than SCORAD (described as a non-objective scale in the evaluation of pruritus) [31]. We heeded this advice in our study. Significant negative effects of skin pruritus on the quality of sleep (prolonged sleep onset latency, decreased sleep time and earlier awakenings) were also observed by Yu et al. [32] in an extensive survey including 5563 adult patients. Also other researchers confirmed (by means of objective methods) the impact of skin lesions severity on the quality of sleep in AD patients $[33,34]$.

Our study was limited by a relatively small number of patients and comparable severity of skin lesions (AD of high and very high severity). We are aware that future studies with more numerous both patients (with a wider spectrum of AD severity) and controls will be of importance. Despite this, significant differences in both groups with varying severity of the disease were revealed. Melatonin concentration was tested only once a day. Evaluating the levels of this neurohormone more frequently increases the accuracy of assays, which may be a suggestion for future studies in this area.

\section{Conclusions}

The severity of skin lesions in AD significantly affects the quality of sleep in patients. Blood melatonin concentration statistically significantly varies in patients with different degrees of skin lesions severity and correlates negatively with the severity of sleep disorders. As the results indicate a significant role of melatonin in the pathophysiology of sleep disorders in patients with $A D$, it would be advisable to continue the research with a larger group of patients.

\section{Conflict of interest}

The authors declare no conflict of interest.

\section{References}

1. Weidinger S, Novak N. Atopic dermatitis. Lancet 2016; 10023: 1109-22.

2. Wollenberg A, Barbarot S, Bieber T, et al. Consensus-based European guidelines for treatment of atopic eczema (atopic dermatitis) in adults and children: part I. J Eur Acad Dermatol Venereol 2018; 32: 657-82.

3. Brunner PM, Silverberg JI, Guttman-Yassky E, et al. Comorbidities suggest that atopic dermatitis is a systemic disorder. J Invest Dermatol 2017; 137: 18-25.

4. Jaworek AK, Jaworek M, Makara-Studzińska M, et al. Depression and serum content of serotonin in adult pa- 
tients with atopic dermatitis. Adv Exp Med Biol 2020 doi: 10.1007/55842019470.

5. Chang YS, Chiang BL. Sleep disorders and atopic dermatitis: a 2-way street? J Allergy Clin Immunol 2018; 142: 1033-40.

6. Gupta MA, Simpson FC, Vujcic B, et al. Obstructive sleep apnea and dermatologic disorders. Clin Dermatol 2017; 35: 319-27.

7. Cicek D, Halisdemir N, Dertioglu SB, et al. Increased frequency of restless legs syndrome in atopic dermatitis. Clin Exp Dermatol 2012; 37: 469-76.

8. Hendricks AJ, Manivannan M, Shi VY. Clinical pearls on sleep management in atopic dermatitis. Dermatitis 2019; 30: 287-93.

9. Hanifin JM, Rajka G. Diagnostic features of atopic dermatitis. Acta Dermatol Venerol (Stockh) 1980; 92: 44-7.

10. Leshem YA, Hajar T, Hanifin JM, et al. What the Eczema Area and Severity Index score tells us about the severity of atopic dermatitis: an interpretability study. Br J Dermatol 2015; 172: 1353-7.

11. Schoch D, Sommer R, Augustin M, et al. Patient-reported outcome measures in pruritus: a systematic review of measurement properties. J Invest Dermatol 2017; 137: 2069-77.

12. Soldatos CR, Dikeos DG, Paparrigopoulos TJ. The diagnostic validity of the Athens Insomnia Scale. J Psychosom Res 2003; 55: 263-7.

13. Barbarot S, Auziere S, Gadkari A, et al. Epidemiology of atopic dermatitis in adults: results from an international survey. Allergy 2018; 73: 1284-93.

14. Smith S, Baker C, Gebauer K, et al. Atopic dermatitis in adults: an Australian management consensus. Australas J Dermatol 2020; 61: 23-32.

15. Raciborski F, Jahnz-Rozyk K, Kłak A, et al. Epidemiology and direct costs of atopic dermatitis in Poland based on the National Health Fund register (2008-2017). Adv Dermatol Allergol 2019; 36: 727-33.

16. Fujii M. Current understanding of pathophysiological mechanisms of atopic dermatitis: interactions among skin barrier dysfunction, immune abnormalities and pruritus. Biol Pharm Bull 2020; 43: 12-9.

17. Li JC, Fishbein A, Singam V, et al. Sleep disturbance and sleep-related impairment in adults with atopic dermatitis: a cross-sectional study. Dermatitis 2018; 29: 270-7.

18. Silverberg J, Garg NK, Paller AS, et al. Sleep disturbances in adults with eczema are associated with impaired overall health: a US population-based study. I Invest Dermatol 2015; 135: 56-66.

19. Chang YS, Chou YT, Lee JH, et al. Atopic dermatitis, melatonin, and sleep disturbance. Pediatrics 2014; 134: 397-405.

20. Camfferman D, Kennedy JD, Gold M, et al. Eczema, sleep, and behavior in children. J Clin Sleep Med 2010; 6: 581-8.

21. Slominski AT, Hardeland R, Zmijewski MA, et al. Melatonin: a cutaneous perspective on its production, metabolism, and functions. J Invest Dermatol 2018; 138: 490-9.

22. Balmik AA, Chinnathambi S. Multi-faced role of melatonin in neuroprotection and amelioration of tau aggregates in Alzheimers disease. J Alzheimers Dis 2018; 63: 1481-93.

23. Harderland R. Melatonin and inflammation - story of a double-edged blade. J Pineal Res 2018; 65: e12525.

24. Schwarz W, Birau N, Hornstein OP, et al. Alterations of melatonin secretion in atopic eczema. Acta Derm Venereol 1988; 68: 224-9.

25. Muńoz-Hoyos A, Espín-Quirantes C, Molina-Carballo A, et al. Neuroendocrine and circadian aspects (melatonin and betaendorphin) of atopic dermatitis in the child. Pediatr Allergy Immunol 2007; 18: 679-86.
26. Chang YS, Lin MH, Lee JH, et al. Melatonin supplementation for children with atopic dermatitis and sleep disturbance: a randomized clinical trial. JAMA Pediatr 2016; 170: 35-42.

27. Taghavi Ardakani A, Farrehi M, Sharif MR, et al. The effects of melatonin administration on disease severity and sleep quality in children with atopic dermatitis: a randomized, double-blinded, placebo-controlled trial. Pediatr Allergy Immunol 2018; 29: 834-40.

28. Marseglia L, Cuppari C, Manti S, et al. Atopic dermatitis: melatonin a potential treatment. J Biol Regul Homeost Agents 2015; 29: 142-9.

29. Patel D, Levoska M, Shwayder T. Managing sleep disturbances in children with atopic dermatitis. Pediatr Dermatol 2018; 35: 428-33.

30. Park G, Lee SH, Oh DS, et al. Melatonin inhibits neuronal dysfunction-associated with neuroinflammation by atopic psychological stress in NC/Nga atopic-like mouse model. J Pineal Res 2017; 63: doi.10.1111/jpi.12420.

31. Kaaz K, Szepietowski JC, Matusiak $Ł$. Influence of itch and pain on sleep quality in atopic dermatitis and psoriasis. Acta Derm Venereol 2019; 99: 175-80.

32. Yu SH, Attarian H, Zee $P$, et al. Burden of sleep and fatigue in US adults with atopic dermatitis. Dermatitis 2016; 27: 50-8.

33. Bender BG, Ballard R, Canono B, et al. Disease severity, scratching, and sleep quality in patients with atopic dermatitis. J Am Acad Dermatol 2008; 58: 415-20.

34. Hon KL, Lam MC, Leung TF, et al. Nocturnal wrist movements are correlated with objective clinical scores and plasma chemokine levels in children with atopic dermatitis. Br J Dermatol 2006; 154: 629-35. 